\title{
A common random fixed point theorem and application to random integral equations
}

\author{
R. A. Rashwan*, D. M. Albaqeri \\ Department of Mathematics, Faculty of Science, Assiut University, Assiut, Egypt \\ *Corresponding author E-mail: rr_rashwan54@yahoo.com
}

Copyright (C)2014 R. A. Rashwan and D. M. Albaqeri. This is an open access article distributed under the Creative Commons Attribution License, which permits unrestricted use, distribution, and reproduction in any medium, provided the original work is properly cited.

\begin{abstract}
The aim of this paper is to prove a fixed point theorem via contraction mappings of a pair of weakly increasing mappings using an altering function in a partially ordered complete separable metric spaces. Our theorem is useful to determine a large of nonlinear problems. We discuss the existence of a common solution to a system of nonlinear random integral equations.
\end{abstract}

Keywords: Common random fixed point, Nonlinear integral random equation, Partially ordered metric spaces, Random variable, Weakly increasing.

\section{Introduction and Preliminary notes}

The application of fixed point theory in different branches of mathematics, statistics, engineering and economics relating to problems associated with approximation theory, theory of differential equations, theory of integral equations, etc. has been recognized in the existing literature [14, 17, 24] and [25]. Random or stochastic integral equations are important in the study of many physical phenomena in life science, engineering and technology $[2,7,14,28,30,31,33,35,36,38,39]$ and $[40]$.

Research of the direction of random fixed points was initiated by Prague School of Probabilistic in connection with random operator theory $[20,21,34]$. Progress in the study on random fixed points of non-expansive mappings, contractive mappings in various spaces has been saturated at large (see for instance) [8]- [13], [15, 24, 27, 29, 37] and many others.

The Banach contraction mapping principle is one of the pivotal results of analysis. There are a lot of the generalizations of the Banach contraction mapping principle in the literature (see e.g.) [1, 3, 16, 19, 32] and others. Khan et al. [26] addressed a new category of contractive fixed point problems for single self-map with the help of a control function that alters distance between two points in a metric space which they called an altering distance.

Definition 1.1 [26] A function $\psi:[0, \infty) \rightarrow[0, \infty)$ is called an altering distance function if the following conditions are satisfied:

1. $\psi(t)=0 \Leftrightarrow t=0$,

2. $\psi$ is a continuous monotonically non-decreasing. 
Recently, Harjani and Sadarangani proved some fixed point theorems for weak contraction and generalized contractions in partially ordered metric spaces by using the altering distance function in $[22,23]$ respectively. Subsequently, Amini-Harandi and Emami proved another fixed point theorem for contraction type maps in partially ordered metric spaces in [5]. After that, Yan et al. [41] extended the results of Harjani and Sadarangani [22, 23] and proved a new fixed point theorem of a contraction mapping principle in a complete metric space endowed with a partially ordered set . They proved the following theorem:

Theorem 1.2 [41] Let $X$ be a partially ordered set and suppose that there exists a metric $d$ in $X$ such that $(X, d)$ is a complete metric space. Let $T: X \rightarrow X$ be a continuous and nondecreasing mapping such that

$\psi(d(T x, T y)) \leq \phi(d(x, y)), \forall x \geq y$,

where $\psi$ is an altering distance function and $\phi:[0, \infty) \rightarrow[0, \infty]$ is a continuous function with the condition $\psi(t)>\phi(t)$ for all $t>0$. If there exists $x_{0} \in X$ such that $x_{0} \leq T x_{0}$, then $T$ has a fixed point.

Let $(X, \Sigma)$ be a separable Banach space, where $\Sigma$ is a $\sigma$-algebra of Borel subsets of $\mathrm{X}$, and $(\Omega, \Sigma, \mu)$ denote a complete probability measure space with measure $\mu$ and $\Sigma$ be a $\sigma$-algebra of subsets of $\Omega$. For more details, see Joshi and Bose [25].

Definition 1.3 A mapping $x: \Omega \rightarrow X$ is called $X$-valued random variable if the inverse image under the mapping $x$ of every Borel set $B$ of $X$ belongs to $\Sigma$, that is, $x^{-1}(B) \in \Sigma$ for all $B \in \Sigma$.

Definition 1.4 A mapping $x: \Omega \rightarrow X$ is said to be a finitely-valued random variable if it is constant on each finite number of disjoint sets $A_{i} \in \Sigma$ and is equal to 0 on $\Omega-\left(\bigcup_{i=1}^{n} A_{i}\right)$. $x$ is called a simple random variable if it is finitely valued and $\mu\{w,\|x(w)\|>0\}<\infty$.

Definition 1.5 A mapping $x: \Omega \rightarrow X$ is said to be a strong random variable if there exists a sequence $\left\{x_{n}(w)\right\}$ of simple random variables which converges to $x(w)$ almost surely, that is, there exists a set $A_{0} \in \Sigma$ with $\mu\left(A_{0}\right)=0$ such that $\lim _{n \rightarrow \infty} x_{n}(w)=x(w), w \in \Omega-A_{0}$.

Definition 1.6 A mapping $x: \Omega \rightarrow X$ is said to be a weak random variable if the function $x^{*}(x(w))$ is a real-valued random variable for each $x^{*} \in X^{*}$, the space $X^{*}$ denoting the first normed dual space of $X$.

Remark 1.7 (1) In a separable Banach space $X$, the notions of strong and weak random variables $x: \Omega \rightarrow X$ coincide, and in respect of such a space $X, x$ is termed as a random variable (see Corollary 1 of Joshi and Bose [25]).

(2) If $X$ is a separable Banach space, then every strong and also weak random variable is measurable in the sense of Definition (1.3).

Let $\mathrm{Y}$ be another Banach space.We also need the following definitions as cited in Joshi and Bose [25].

Definition 1.8 Amapping $F: \Omega \times X \rightarrow Y$ is said to be a random mapping if $F(w, x)=Y(w)$ is a $Y$-valued random variable for every $x \in X$..

Definition 1.9 A mapping $F: \Omega \times X \rightarrow Y$ is said to be a continuous random mapping if the set of all $w \in \Omega$ for which $F(w, x)$ is a continuous function of $x$ has measure one.

Definition 1.10 An equation of the type $F(w, x(w))=x(w)$, where $F: \Omega \times X \rightarrow X$ is a random mapping, is called a random fixed point equation.

Definition 1.11 Any mapping $x: \Omega \rightarrow X$ which satisfies the random fixed point equation $F(w, x(w))=x(w)$ almost surely is said to be a wide sense solution of the fixed point equation.

Definition 1.12 Any X-valued random variable $x(w)$ which satisfies $\mu\{w: F(w, x(w))=x(w)\}=1$ is said to be a random solution of the fixed point equation or a random fixed point of $F$.

Remark 1.13 A random solution is a wide sense solution of the fixed point equation, but the converse is not necessarily true. This is evident from the example as found under Remark 1 in the work of Joshi and Bose [25].

In this paper, we extend the result of Yan et al. [41] and many others for a pair weakly increasing random mappings satisfying a new contraction in a partially ordered metric space. As application, we establish the existence of a common solution to system of nonlinear random integral equations. 


\section{A common random fixed point theorem of weakly increasing map- pings}

We need the following notions in our work:

Definition 2.1 $[18,4]$ Let $(X, \leq)$ be a partially ordered set and $f$ and $g$ be two self maps on $X$. An ordered pair $(f, g)$ is said to be weakly increasing if $f x \leq g f x$ and $g x \leq f g x$ for all $x \in X$.

Lemma 2.2 (see [41]) If $\psi$ is an altering distance function and $\phi:[0, \infty) \rightarrow[0, \infty)$ is a continuous function with the condition $\psi(t)>\phi(t)$ forallt $>0$, then $\phi(0)=0$.

Now, we state our main results:

Theorem 2.3 Let $(X, \leq)$ be a partially ordered set and suppose that there exists a metric $d$ in $X$ such that $(X, d)$ is a complete separable metric space and $(\Omega, \Sigma, \mu)$ is a complete probability measure space. Let $T, S: \Omega \times X \rightarrow X$ be two mappings such that

(i) $T(w,$.$) and S(w,$.$) are continuous mappings for all w \in \Omega$;

(ii) $T(., x)$ and $S(., y)$ are measurable mappings for all $x, y, \in X$;

(iii) The pair $(T, S)$ is weakly increasing such that for all comparable $x$ and $y$ we have

$\psi(d(T(w, x), S(w, y))) \leq \phi(d(x, y)), w \in \Omega$

where $\psi$ is an altering distance function and $\phi:[0, \infty) \rightarrow[0, \infty]$ is a continuous function with the condition

$\psi(t)>\phi(t)$, forall $t>0$,

also suppose either

(a) $T$ or $S$ is continuous or

(b) $X$ has the following property:

If a non-decreasing sequence $\left\{x_{n}\right\} \rightarrow x$ then $x_{n} \leq x$ for all $n$,

then $T$ and $S$ have a common random fixed point.

Proof. Let the function $\xi_{0}(w): \Omega \rightarrow X$ be an arbitrary measurable mapping. We can define a sequence of measurable mappings $\left\{\xi_{n}(w)\right\}$ from $\Omega$ to $X$ as following:

$\xi_{2 n+1}(w)=T\left(w, \xi_{2 n}(w)\right)$,

$\xi_{2 n+2}(w)=S\left(w, \xi_{2 n+1}(w)\right), w \in \Omega, n=0,1,2, \ldots$.

Since the pair $(T, S)$ is weakly increasing mappings, we have

$\xi_{1}(w)=T\left(w, \xi_{0}(w)\right) \leq S\left(w, T\left(w, \xi_{0}(w)\right)\right)=S\left(w, \xi_{1}(w)\right)=\xi_{2}(w)$,

$\xi_{2}(w)=T\left(w, \xi_{1}(w)\right) \leq S\left(w, T\left(w, \xi_{1}(w)\right)\right)=S\left(w, \xi_{2}(w)\right)=\xi_{3}(w)$,

Continuing this process, we get

$\begin{aligned} \xi_{2 n+1}(w) & =T\left(w, \xi_{2 n}(w)\right) \leq S\left(w, T\left(w, \xi_{2 n}(w)\right)\right)=S\left(w, \xi_{2 n+1}(w)\right)=\xi_{2 n+2}(w), \\ \text { and } & \\ \xi_{2 n+2}(w) & =S\left(w, \xi_{2 n+1}(w)\right) \leq T\left(w, S\left(w, \xi_{2 n+1}(w)\right)\right)=T\left(w, \xi_{2 n+2}(w)\right)=\xi_{2 n+3}(w) .\end{aligned}$

Thus for all $n \geq 1$, we have $\xi_{n}(w) \leq \xi_{n+1}(w)$

Without loss of the generality, we can assume that $\xi_{n}(w) \neq \xi_{n+1}(w)$ and since $\xi_{2 n}(w)$ and $\xi_{2 n+1}(w)$ are comparable, then by (1), we obtain

$\begin{aligned} \psi\left(d\left(\xi_{2 n+1}(w), \xi_{2 n+2}(w)\right)\right) & =\psi\left(d\left(T\left(w, \xi_{2 n}(w)\right), S\left(w, \xi_{2 n+1}(w)\right)\right)\right) \\ & \leq \phi\left(d\left(\xi_{2 n}(w), \xi_{2 n+1}(w)\right)\right),\end{aligned}$ 
using the condition (2) and since $\psi$ non-decreasing, we get

$\left.d\left(\xi_{2 n+1}(w), \xi_{2 n+2}(w)\right)\right) \leq d\left(\xi_{2 n}(w), \xi_{2 n+1}(w)\right)$.

Similarly, we can show that

$\left.d\left(\xi_{2 n+2}(w), \xi_{2 n+3}(w)\right)\right) \leq d\left(\xi_{2 n+1}(w), \xi_{2 n+2}(w)\right)$.

From (5) and (6), we get

$\left.d\left(\xi_{n+1}(w), \xi_{n+2}(w)\right)\right) \leq d\left(\xi_{n}(w), \xi_{n+1}(w)\right)$.

Hence, $\left.\left\{d\left(\xi_{n}(w), \xi_{n+1}(w)\right)\right)\right\}$ is a nonnegative decreasing sequence, then there exists $r(w) \geq 0$ such that

$\left.\lim _{n \rightarrow \infty} d\left(\xi_{n}(w), \xi_{n+1}(w)\right)\right)=r(w), w \in \Omega$.

Letting $n \rightarrow \infty$ in (4)

$\psi(r(w)) \leq \phi(r(w))$,

by the condition (2), we get $r(w)=0, w \in \Omega$, thus

$\left.\lim _{n \rightarrow \infty} d\left(\xi_{n}(w), \xi_{n+1}(w)\right)\right)=0, w \in \Omega$.

Next, we will show that for $w \in \Omega,\left\{\xi_{n}(w)\right\}$ is a Cauchy sequence in $X$, it is sufficient to prove that $\left\{\xi_{2 n}(w)\right\}$ is a Cauchy sequence. We proceed by negation, suppose that $\left\{\xi_{2 n}(w)\right\}$ is not a Cauchy sequence, then there exists $\epsilon>0$ for which we can find two sequences of positive integers $\{m(k)\},\{n(k)\}$ such that for positive integer $k$ we have

$m(k)>n(k)>k, d\left(\xi_{2 n(k)}(w), \xi_{2 m(k)}(w)\right) \geq \epsilon, k \geq 1$.

Further, we can choose $m_{k}$ to be smallest integer with $m(k)>n(k)$ for which (10) holds. Then

$d\left(\xi_{2 n(k)}(w), \xi_{2 m(k)-2}(w)\right)<\epsilon$.

From (10), (11) and the triangle inequality, we obtain

$$
\begin{aligned}
\epsilon \leq d\left(\xi_{2 n(k)}(w), \xi_{2 m(k)}(w)\right) & \leq d\left(\xi_{2 n(k)}(w), \xi_{2 m(k)-2}(w)\right)+d\left(\xi_{2 m(k)-2}(w), \xi_{2 m(k)-1}(w)\right) \\
& +d\left(\xi_{2 m(k)-1}(w), \xi_{2 m(k)}(w)\right) \\
& <\epsilon+d\left(\xi_{2 m(k)-2}(w), \xi_{2 m(k)-1}(w)\right) \\
& +d\left(\xi_{2 m(k)-1}(w), \xi_{2 m(k)}(w)\right),
\end{aligned}
$$

Letting $k \rightarrow \infty$ in the above inequality and using (9) we have

$\lim _{n \rightarrow \infty} d\left(\xi_{2 n(k)}(w), \xi_{2 m(k)}(w)\right)=\epsilon, w \in \Omega$.

In addition, by the triangle inequality, we have

$$
\begin{aligned}
d\left(\xi_{2 n(k)}(w), \xi_{2 m(k)}(w)\right) & \leq d\left(\xi_{2 n(k)}(w), \xi_{2 n(k)-1}(w)\right)+d\left(\xi_{2 n(k)-1}(w), \xi_{2 m(k)-1}(w)\right) \\
& +d\left(\xi_{2 m(k)-1}(w), \xi_{2 m(k)}(w)\right)
\end{aligned}
$$

and

$$
\begin{aligned}
d\left(\xi_{2 n(k)-1}(w), \xi_{2 m(k)-1}(w)\right) & \leq d\left(\xi_{2 n(k)-1}(w), \xi_{2 n(k)}(w)\right)+d\left(\xi_{2 n(k)}(w), \xi_{2 m(k)}(w)\right) \\
& +d\left(\xi_{2 m(k)}(w), \xi_{2 m(k)-1}(w)\right)
\end{aligned}
$$

Letting $k \rightarrow \infty$ in the above two inequalities, using (9) and (12) we obtain

$\lim _{n \rightarrow \infty} d\left(\xi_{2 n(k)-1}(w), \xi_{2 m(k)-1}(w)\right)=\epsilon$.

Since $m(k)>n(k)$ and $\xi_{2 n(k)-1}(w)$ and $\xi_{2 m(k)-1}(w)$ are comparable, then by (1), we obtain

$$
\begin{aligned}
\psi\left(d\left(\xi_{2 n(k)}(w), \xi_{2 m(k)}(w)\right)\right) & =\psi\left(d\left(T\left(w, \xi_{2 n(k)-1}(w)\right), S\left(w, \xi_{2 m(k)-1}(w)\right)\right)\right) \\
& \leq \phi\left(d\left(\xi_{2 n(k)-1}(w), \xi_{2 m(k)-1}(w)\right)\right),
\end{aligned}
$$


Letting $k \rightarrow \infty$, using (12) and (13), we get

$\psi(\epsilon) \leq \phi(\epsilon)$,

By the condition (2), we obtain $\epsilon=0$, which is a contradiction. It follows that $\left\{\xi_{2 n}\right\}$ is a Cauchy sequence in $X$ and so is $\left\{\xi_{n}\right\}$, then there exists $\xi(w): \Omega \rightarrow X$ such that

$\lim _{n \rightarrow \infty} \xi_{n}(w)=\xi(w), w \in \Omega$,

Now, if $T$ is continuous, then

$\xi(w)=\lim _{n \rightarrow \infty} \xi_{2 n+1}(w)=\lim _{n \rightarrow \infty} T\left(w, \xi_{2 n}(w)\right)=T(w, \xi(w))$,

also since $\xi(w) \leq \xi(w)$, using (1), (14) and Lemma 2.2, we obtain

$$
\begin{aligned}
\psi(d(\xi(w), S(w, \xi(w)))) & =\psi(d(T(w, \xi(w)), S(w, \xi(w)))) \\
& \leq \phi(d(\xi(w), \xi(w)))=0 .
\end{aligned}
$$

Thus

$\xi(w)=S(w, \xi(w))$.

From (14) and (15), we obtain

$\xi(w)=T(w, \xi(w))=S(w, \xi(w))$.

Similarly, we obtain the same result if $S$ is continuous.

Now, if the condition (b) is satisfied, since $\lim _{n \rightarrow \infty} \xi_{2 n-1}(w)=\xi(w)$, then by the condition (b), we have $\xi_{2 n-1}(w) \leq$ $\xi(w)$, thus we have by $(1)$

$$
\begin{aligned}
\psi\left(d\left(\xi_{2 n}(w), S(w, \xi(w))\right)\right) & =\psi\left(d\left(T\left(w, \xi_{2 n-1}(w)\right), S(w, \xi(w))\right)\right) \\
& \leq \phi\left(d\left(\xi_{2 n-1}(w), \xi(w)\right)\right)
\end{aligned}
$$

Letting $k \rightarrow \infty$, and using Lemma 2.2, then we have $\xi(w)=S(w, \xi(w))$. Similarly, we can show that $\xi(w)=$ $T(w, \xi(w))$.

The proof of the theorem is completed.

Taking $T=S$ in Theorem 2.3, we obtain the following Corollary:

Corollary 2.4 Let $(X, \leq)$ be a partially ordered set and suppose that there exists a metric $d$ in $X$ such that $(X, d)$ is a complete separable metric space and $(\Omega, \Sigma, \mu)$ is a complete probability measure space. Let $T: \Omega \times X \rightarrow X$ be such that

(i) $T(w,$.$) is continuous mapping for w \in \Omega$;

(ii) $T(., x)$ is measurable mapping for $x \in X$;

(iii) $T$ is a non-decreasing mapping such that $\xi_{0}(w) \leq T\left(w, \xi_{0}(w)\right), w \in \Omega$ for some measurable mappings $\xi_{0}(w)$ in $X$, and for all comparable $x$ and $y$ we have

$\psi(d(T(w, x), T(w, y))) \leq \phi(d(x, y)), w \in \Omega$,

where $\psi$ is an altering distance function and $\phi:[0, \infty) \rightarrow[0, \infty]$ is a continuous function with the condition

$\psi(t)>\phi(t)$, forall $t>0$,

also suppose either

(a) $T$ is continuous or

(b) $X$ has the following property:

If a non-decreasing sequence $\left\{x_{n}\right\} \rightarrow x$ then $x_{n} \leq x$ for all $n$,

then $T$ has a random fixed point.

Remark 2.5 In Corollary 2.4, a sufficient condition for the uniqueness of the fixed point is: for two measurable mappings $\eta(w), \zeta(w): \Omega \rightarrow X$ there exists a measurable mapping $\xi(w): \Omega \rightarrow X$ which is comparable with both $\eta(w)$ and $\zeta(w)$. 


\section{Application}

In this section, we give an application of Theorem 2.3 to prove the existence of a common solution to the following random nonlinear integral equations:

$x(t ; w)=h(t ; w)+\int_{S} k_{1}(t, s ; w) f_{1}(s, x(s ; w)) d \mu(s)$
$x(t ; w)=h(t ; w)+\int_{S} k_{2}(t, s ; w) f_{2}(s, x(s ; w)) d \mu(s)$,

where

(i) $\mathrm{S}$ is a locally compact metric space with a metric d on $S \times S$ equipped with a complete $\sigma$-finite measure $\mu$ defined on the collection of Borel subsets of S;

(ii) $w \in \Omega$, the supporting set of the probability measure space $(\Omega, \Sigma, \mu)$;

(iii) $h(t ; w)$ are stochastic free terms defined for $t \in S$;

(iv) $x(t ; w)$ is the unknown vector valued random variable for $t \in S$;

(v) $k_{i}(t, s ; w), i=1,2$ are stochastic kernels defined for $t$ and $s$ in $\mathrm{S}$ with $k_{1}=k_{2}=k$ and

(vi) $f_{i}(s, x), i=1,2$ are vector-valued functions for all $s \in S$ and $x$.

The integrals in the two equations (16) are interpreted as Bochner integrals [42].

We will further assume that $\mathrm{S}$ is the union of a countable family of compact subsets $\left\{C_{n}\right\}$ having the properties that $C_{1} \subset C_{2} \subset \ldots$ and that for any other compact set $S$ there is a $C_{i}$ which contains it [6].

We will follow the steps of Lee and Padgett [27] with necessary modifications as required for the more general settings.

Definition 3.1 We define the space $C\left(S, L_{2}(\Omega, \Sigma, \mu)\right)$ to be the space of all continuous functions from $S$ into $L_{2}(\Omega, \Sigma, \mu)$ with the topology of uniform convergence on compact sets of $S$, that is, for each fixed $t \in S, x(t ; w)$ is a vector-valued random variable such that

$\|x(t ; w)\|_{L_{2}(\Omega, \Sigma, \mu)}^{2}=\int_{\Omega}|x(t ; w)|^{2} d \mu(w)<\infty$.

Note that $C\left(S, L_{2}(\Omega, \Sigma, \mu)\right)$ is locally convex space (see [42]) whose topology is defined by a countable family of semi-norms given by

$\|x(t ; w)\|_{n}=\sup _{t \in C_{n}}\|x(t ; w)\|_{L_{2}(\Omega, \Sigma, \mu)}, n=1,2, \ldots$

Moreover, $C\left(S, L_{2}(\Omega, \Sigma, \mu)\right)$ is complete relative to this topology since $L_{2}(\Omega, \Sigma, \mu)$ is complete.

We will consider the functions $h(t ; w)$ and $f_{i}(t, x(t ; w)), i=1,2$ to be in the space $C\left(S, L_{2}(\Omega, \Sigma, \mu)\right)$ with respect to the stochastic kernel. We assume that for each pair $(t, s), k_{i}(t, s ; w) \in L_{\infty}(\Omega, \Sigma, \mu)$ and denote the norm by

$\||k(t, s ; w)|\|=\|k(t, s ; w)\|_{L_{\infty}(\Omega, \Sigma, \mu)}=\mu-e s s \sup _{w \in \Omega}|k(t, s ; w)|$.

Also, we will suppose that $k(t, s ; w)$ is such that $\left\|\left|k(t, s ; w)\|\mid \cdot\| x(t ; w) \|_{L_{2}(\Omega, \Sigma, \mu)}\right.\right.$ is $\mu$-integrable with respect to s for each $t \in S$ and $x(s ; w)$ in $C\left(S, L_{2}(\Omega, \Sigma, \mu)\right)$ and that there exists a real-valued function $\mathrm{G}$ defined $\mu$-a.e. on $\mathrm{S}$ so that $G(s)\|x(s ; w)\|_{L_{2}(\Omega, \Sigma, \mu)}$ is $\mu$-integrable and, for each pair $(t, s) \in S \times S$,

$|\|k(t, u ; w)-k(s, u ; w)\|| \cdot\|x(t ; w)\|_{L_{2}(\Omega, \Sigma, \mu)} \leq G(u)\|x(t ; w)\|_{L_{2}(\Omega, \Sigma, \mu)}$

$\mu$-a.e. Further, for almost all $s \in S, k(t, s ; w)$ will be continuous in t from $\mathrm{S}$ into $L_{\infty}(\Omega, \Sigma, \mu)$.

We now define the integral operator $(\mathrm{A})$ on $C\left(S, L_{2}(\Omega, \Sigma, \mu)\right)$ by

$(A x)(t ; w)=\int_{S} k(t, s ; w) x(s ; w) d \mu(s)$,

where the integral is Bochner integral. From the conditions on $k(t, s ; w)$, we have that for each $t \in S,(A x)(t ; w) \in$ $L_{2}(\Omega, \Sigma, \mu)$ and that $(A x)(t ; w)$ is continuous in mean square by Lebesgue's dominated convergence theorem. That is, $(A x)(t ; w) \in C\left(S, L_{2}(\Omega, \Sigma, \mu)\right)$. 
Definition 3.2 [27, 30] Let $B$ and $D$ be Banach spaces. The pair $(B, D)$ is said to be admissible with respect to a linear operator $A(w)$ if $A(w)(B) \subset D$.

Lemma 3.3 (see [30]) The linear operator (A) defined by equation (17) is continuous from $C\left(S, L_{2}(\Omega, \Sigma, \mu)\right)$ into itself.

Lemma 3.4 (see [38, 30]) If $(A)$ is a continuous linear operator from $C\left(S, L_{2}(\Omega, \Sigma, \mu)\right)$ into itself and $B, D \subset$ $C\left(S, L_{2}(\Omega, \Sigma, \mu)\right)$ are Banach spaces stronger than $C\left(S, L_{2}(\Omega, \Sigma, \mu)\right)$ such that $(B, D)$ is admissible with respect to $A$, then $(A)$ is continuous from $B$ into $D$.

Remark 3.5 (1) From Lemmas 3.3 and 3.4, it follows that the operator (A) defined by equation (17) is a bounded linear operator from $B$ into $D$.

(2) By a common random solution of the equations (16) we will mean a function $x(t ; w)$ in $C\left(S, L_{2}(\Omega, \Sigma, \mu)\right)$ which satisfies the equations $\mu$-a.e.

We now present theorem concerning the existence of a common random solution of the equations (16).

Theorem 3.6 Consider the stochastic nonlinear integral equations (16) equipped with the following conditions:

(a) $B$ and $D$ are Banach spaces stronger than $C\left(S, L_{2}(\Omega, \Sigma, \mu)\right)$ such that $(B, D)$ is admissible with respect to the integral operator $A$ defined by (17).

(b) $x(t ; w) \rightarrow f_{i}(t, x(t ; w)), i=1,2$ are operator from the set

$$
Q(\rho)=\left\{x(t ; w): x(t ; w) \in D,\|x(t ; w)\|_{D} \leq \rho\right\}
$$

into the space $B$ satisfying the following:

$$
\begin{aligned}
& \left\|f_{1}(t, x(t ; w))-f_{1}(t, y(t ; w))\right\|_{B} \leq \alpha(w)\|x(t ; w)-y(t ; w)\|_{D} \\
& \left\|f_{2}(t, x(t ; w))-f_{2}(t, y(t ; w))\right\|_{B} \leq \beta(w)\|x(t ; w)-y(t ; w)\|_{D} \\
& \left\|f_{1}(t, x(t ; w))-f_{2}(t, y(t ; w))\right\|_{B} \leq \gamma(w)\|x(t ; w)-y(t ; w)\|_{D}
\end{aligned}
$$

for $x(t ; w), y(t ; w) \in Q(\rho)$, where $\rho$ is a constant and $\alpha(w), \beta(w), \gamma(w)$ are real-valued random variables.

(c) $h(t ; w) \in D$

(d) $f_{1}(s, x(s ; w)) \leq f_{2}\left(s, h(t ; w)+\int_{S} k(t, \tau ; w) f_{1}(\tau, x(\tau ; w)) d \mu(\tau)\right)$ and

(e) $f_{2}(s, x(s ; w)) \leq f_{1}\left(s, h(t ; w)+\int_{S} k(t, \tau ; w) f_{2}(\tau, x(\tau ; w)) d \mu(\tau)\right)$.

Then, the system of the stochastic nonlinear integral equations (16) has a common solution in $Q(\rho)$ provided $\alpha(w) \lambda(w)<1, \beta(w) \lambda(w)<1$ and $\gamma(w) \lambda(w)<1$ and

$\|h(t ; w)\|_{D}+k\left\|f_{1}(t, 0)\right\|_{B} \leq \rho(1-\alpha(w) \lambda(w))$,

$\|h(t ; w)\|_{D}+k\left\|f_{2}(t, 0)\right\|_{B} \leq \rho(1-\beta(w) \lambda(w))$,

where $K$ is the norm of the operator $A$.

Prrof. Define the operators $U$ and $V$ from $Q(\rho)$ into D by

$(U x)(t ; w)=h(t ; w)+\int_{S} k(t, s ; w) f_{1}(s, x(s ; w)) d \mu(s)$
$(V x)(t ; w)=h(t ; w)+\int_{S} k(t, s ; w) f_{2}(s, x(s ; w)) d \mu(s)$.

Then from the conditions of this theorem

$$
\begin{aligned}
\|(U x)(t ; w)\|_{D} & \leq\|h(t, w)\|_{D}+\int_{S}\left\|k(t, s, w) f_{1}(s, x(s, w)) d \mu(s)\right\| \\
& \leq\|h(t ; w)\|_{D}+\lambda(w)\left\|f_{1}(t, x(t, w))\right\|_{B} \\
& \leq\|h(t ; w)\|_{D}+\lambda(w)\|f(t, 0)\|_{B}+\lambda(w)\left\|f_{1}(t, x(t ; w))-f_{1}(t, 0)\right\|_{B}
\end{aligned}
$$


Using the first condition of (18) and (19), we get

$$
\begin{aligned}
\|(U x)(t ; w)\|_{D} & \leq\|h(t ; w)\|_{D}+\lambda(w)\left\|f_{1}(t, 0)\right\|_{B}+\lambda(w) \alpha(w)\|x(t ; w)\|_{D} \\
& \leq \rho(1-\alpha(w) \lambda(w))+\alpha(w) \lambda(w) \rho=\rho
\end{aligned}
$$

Hence $(U x)(t ; w) \in Q(\rho)$. Similarly, by using the second condition of (18) and (19), we can prove that $(V x)(t ; w) \in$ $Q(\rho)$.

Next, we claim that the pair $(U, V)$ is weakly increasing. Indeed, by the condition $(\mathrm{d})$, we get

$$
\begin{aligned}
(U x)(t ; w) & =h(t ; w)+\int_{S} k(t, s ; w) f_{1}(s, x(s ; w)) d \mu(s) \\
& \leq h(t, w)+\int_{S} k(t, s, w) f_{2}\left(s, h(t, w)+\int_{S} k(t, \tau ; w) f_{1}(\tau, x(\tau ; w)) d \mu(\tau)\right) d \mu(s) \\
& =h(t ; w)+\int_{S} k(t, s ; w) f_{2}(s,(U x)(t ; w)) d \mu(s) \\
& =(V U x)(t ; w) .
\end{aligned}
$$

Similarly, by condition (e), we can show that $(V x)(t ; w) \leq(U V x)(t ; w)$.

Now, for $x(t ; w), y(t ; w) \in Q(\rho)$ we see by the third condition of $(18)$ that

$$
\begin{aligned}
\|(U x)(t ; w)-(V y)(t ; w)\|_{D} & =\left\|\int_{S} k(t, s ; w)\left[f_{1}(s, x(s ; w))-f_{2}(s, y(s ; w))\right] d \mu(s)\right\|_{D} \\
& \leq \lambda(w)\left\|f_{1}(s, x(s ; w))-f_{2}(s, y(s ; w))\right\|_{B} \\
& \leq \lambda(w) \gamma(w)\|x(t ; w)-y(t ; w)\|_{D} .
\end{aligned}
$$

Putting $\psi(x)=x$ and $\phi(x)=\lambda(w) \gamma(w) x$, then clearly $\psi$ is an altering distance function and $\phi$ is continuous function satisfying $\psi(x)>\phi(x)$ for $x>0$ and $\lambda(w) \gamma(w)<1$. From (20), we obtain for comparable $x, y$ that

$$
\psi\left(\|(U x)(t ; w)-(V y)(t ; w)\|_{D}\right) \leq \phi\left(\|x(t ; w)-y(t ; w)\|_{D}\right) .
$$

Hence all the required hypotheses of Theorem 2.3 are satisfied. Then $U$ and $V$ have a common random fixed point $x^{*}(t ; w)$ which is a stochastic solution to the equations (16).

\section{Conclusion}

Applying Theorem 2.3, we can obtain a common solution to the equations (16) under certain conditions. Consequently, we can also applying Corollary 2.4 to obtain a solution to a nonlinear random integral equation

$$
x(t ; w)=h(t ; w)+\int_{S} k(t, s ; w) f(s, x(s ; w)) d \mu(s),
$$

with some similar conditions to Theorem 3.6.

\section{Acknowledgements}

The authors would like to thank the editor of International Journal of Applied Mathematical Research for his support.

\section{References}

[1] M. Abbas and G. Jungck, "Common fixed point results for noncommuting mappings without continuity in cone metric spaces", Journal of Mathematical Analysis and Applications, Vol.341, (2008), 416-420.

[2] J. Achari, "On a pair of random generalized nonlinear contractions", Int. J. Math. Math. Sci., Vol.6, No.3, (1983), 467-475.

[3] Ya. I. Alber, S. Guerre-Delabriere, "Principle of weakly contractive maps in Hilbert spaces", Oper. Theory Adv. Appl., Vol.98, (1997), 7-22. 
[4] I. Altun and H. Simsek, "Some fixed point theorems on ordered metric spaces and application", Fixed Point Theory and Applications, Vol.2010, (2010), 1-17. Article ID 621469.

[5] A. Amini-Harandi, H. Emami, "A fixed point theorem for contraction type maps in partially ordered metric spaces and application to ordinary differential equations", Nonlinear Anal., Vol.72, (2010), 2238-2242.

[6] R. F. Arens, "A topology for spaces of transformations", Anl. Math., Vol.47, No.2, (1946), 480-495.

[7] K. Balachandran and J. H. Kim, "Existence of solutions of nonlinear stochastic Volterra Fredholm integral equations of mixed type", International Journal of Mathematics and Mathematical sciences, Vol.2010, (2010) , 1-16. Artical ID 603819 doi:10.1155/2010/603819.

[8] I. Beg, "Random fixed points of random operators satisfying semicontractivity conditions", Math. Japan., Vol.46, (1997), 151-155.

[9] I. Beg, "Approximaton of random fixed points in normed spaces", Nonlinear Anal., Vol.51, (2002), 1363-1372.

[10] I. Beg, "Random Coincidence and fixed points for weakly compatible mappings in convex metric spaces", Asian-European Journal of Mathematics, Vol.2, (2009), 171-182.

[11] I. Beg, M. Abbas, "Common random fixed points of compatible random operators", Int. J. Math. and Mathematical Sciences, Vol.2006, (2006), 1-15.

[12] I. Beg, N. Shahzad, "Random fixed point theorems on product spaces", J. Appl. Math. Stochastic Anal., Vol.6, (1993), 95-106.

[13] I. Beg, N. Shahzad, "Random fixed point theorems for nonepansive and contractive type random operators on Banach spaces", J. Appl. Math. Stochastic Anal., Vol.7, (1994), 569-580.

[14] A. T. Bharucha-Reid, "Random Integral Equations, Mathematics in Science and Engineering", Academic Press, New York, NY, USA, Vol.9, (1972).

[15] A.T. Bharucha-Reid, "Fixed point theorems in probabilistic analysis", Bull. Amer. Math. Soc., Vol.82, (1976), 641-65.

[16] B. S. Choudhury, "A common unique fixed point result in metric spaces involving generalized altering distances", Math. Commun., Vol.10, (2005), 105-110.

[17] L. Debnath, P. Mikusinski, "Introduction to Hilbert Spaces with Application", Academic Press, Boston (2005).

[18] B. C. Dhage, "Condensing mappings and applications to existence theorems for common solution of differential equations", Bull. Korean Math. Soc., Vol.36, No.3, (1999), 565-578.

[19] P. N. Dutta, B. S. Choudhury, "A generalisation of contraction principle in metric spaces", Fixed Point Theory Appl., Vol.2008, (2008), 1-8. Article ID 406368.

[20] O. Hans, "Reduzierende zulliallige transformaten", Czechoslovak Math. J., Vol.7, (1957), 154-158.

[21] O. Hans, "Random operator equations", Proceedings of the fourth Berkeley Symposium on Math., Statistics and Probability II, PartI, (1961), 85-202.

[22] J. Harjani, k. Sadarangni, "Fixed point theorems for weakly contraction mappings in partially ordered sets", Nonlinear Anal., Vol.71, (2009), 3403-3410.

[23] J. Harjani, k. Sadarangni, "Generalized contractions in partially ordered metric spaces and applications to ordinary differential equations", Nonlinear Anal., Vol.72, (2010), 1188-1197.

[24] S. Itoh, "Random fixed point theorems with an application to random differential equations in Banach spaces", J. Math. Anal. Appl., Vol.67, (1979), 261-273.

[25] MC. Joshi, RK. Bose, "Some Topics in Nonlinear Functional Analysis", Wiley, New York, (1984).

[26] M. S. Khan, M. Swaleh, S. Sessa, "Fixed point theorems by altering distances between the points", Bull. Aust. Math. Soc., Vol.30, No.1, (1984), 1-9.

[27] A. C. H. Lee and W. J. Padgett, "On random nonlinear contraction", Math. Systems Theory, Vol.II, (1977), 77-84.

[28] A. C. H. Lee and W. J. Padgett, "A random nonlinear integral equation in population growth problems", Journal of Integral Equations, Vol.2, No.1, (1980), 1-9. 
[29] A. Mukherjee, "Random transformations of Banach spaces", Ph. D. Dissertation, Wayne State University, Detroit, Machigan, (1986).

[30] W. J. Padgett, "On a nonlinear stochastic integral equation of the Hammerstein type", Proc. Amer. Math. Soc., Vol.38, No.1, (1973), 625-631.

[31] W. J. Padgett and C. P. Tsokos, "A random Fredholm integral equation", Proceedings of the American Mathematical Society, Vol.33, (1972), 534-542.

[32] B. E. Rhoades, "Some theorems on weakly contractive maps", Nonlinear Anal., Vol.47, No.4, (2001), $2683-2693$.

[33] M. Saha and D. Dey, "Some random fixed point theorems for $(\theta, L)$-weak contractions", Hacettepe Journal of Mathematics and Statistics, Vol.41, No.6, (2012), 795-812.

[34] A. Spacek, "Zufallige gleichungen", Czechoslovak Math. J., Vol.5, (1955), 462-466.

[35] R. Subramaniam, K. Balachandran, and J. K. Kim, "Existence of solutions of a stochastic integral equation with an application from the theory of epidemics", Nonlinear Functional Analysis and Applications, Vol.5, No.1, (2000), 23-29.

[36] D. Szynal and S. Wedrychowicz, "On solutions of a stochastic integral equation of the Volterra type with applications for chemotherapy", Journal of Applied Probability, Vol.25, No.2, (1988), 257-267.

[37] K. K. Tan, X.Z. Yuan, "Random fixed-point theorems and approximation in cones", J. Math. Anal. Appl., Vol.185, No.2, (1994), 378-390.

[38] C. P. Tsokos, "On a stochastic integral equation of the Volterra type", Math. Systems Theory, Vol.3, (1969), 222-231.

[39] C. P. Tsokos and W. J. Padgett, "Random Integral Equations with Applications to Stochastic Sytems", Lecture Notes in Mathematics, Springer, Berlin, Germany, Vol.233, (1971).

[40] C. P. Tsokos and W. J. Padgett, "Random Integral Equations with Applications to Life Sciences and Engineering", Academic Press, New York, 1974.

[41] F. Yan, Y. Su, Q. Feng, "A new contaction mapping principle in partially ordered metric spaces and applications to ordinary differential equations", Springer Open J., Vol.152, (2012), 1-13.

[42] K. Yosida, "Functional Analysis", Springer, Berlin (1965). 